Rapid Reviews COVID-19

\title{
Review 2: "Neutralization of N501Y mutant SARS- CoV-2 by BNT162b2 vaccine-elicited sera"
}

Kimberly Luke ${ }^{1}$

${ }^{1}$ Intuitive Biosciences, Research and Development, USA

Published on: Feb 07, 2021

License: Creative Commons Attribution 4.0 International License (CC-BY 4.0). 


\section{$\underline{\text { RR:C19 Evidence Scale rating by reviewer: }}$}

- Potentially informative. The main claims made are not strongly justified by the methods and data, but may yield some insight. The results and conclusions of the study may resemble those from the hypothetical ideal study, but there is substantial room for doubt. Decision-makers should consider this evidence only with a thorough understanding of its weaknesses, alongside other evidence and theory. Decision-makers should not consider this actionable, unless the weaknesses are clearly understood and there is other theory and evidence to further support it.

\section{Review:}

This manuscript provides a very brief summary of a single experiment testing the ability of sera from BNT162b2 vaccinated individuals to neutralize SARS-CoV-2 viruses, with a focus on comparing the wild type USA-WA1/2020 strain (N501) and a lab-generated strain. The lab generated strain encodes the Y501 spike mutant, which is one of the key mutations in the rapidly spreading variants arising in the United Kingdom and South Africa. The authors present only one figure showing the plaque reduction neutralization titer of the N501 strain and compares to the Y501 strain, which the authors conclude has little to no significant difference. While this is interesting data, the manuscript is lacking discussion and further investigation. This manuscript seems incomplete. Further experiments testing efficacy of the BNT162b2 vaccine to prevent infection in a model system would be important. Even a discussion, if not experimentation, around their mutant strain would be important for this manuscript to be considered a full work for publication. Does their lab generated strain replicate to similar levels as their WT strain? The general claim that there is "Neutralization of N501Y mutant SARS-CoV-2 by BNT162b2 vaccine-elicited sera" seems weakly supported by only one experiment. It is not misleading, but very limited in its evaluation. Are there other mutations, beyond the N501Y in the spike protein, that are important to viral infectivity? If this N501Y mutation is key to increasing the host range to mice, it seems critical to show that the BNT162b2 provides protection to mice when challenges with this mutant. Overall, this submission seems incomplete and not a full manuscript. 